\title{
ENABLING LONGITUDINAL DATA COMPARISON USING DDI
}

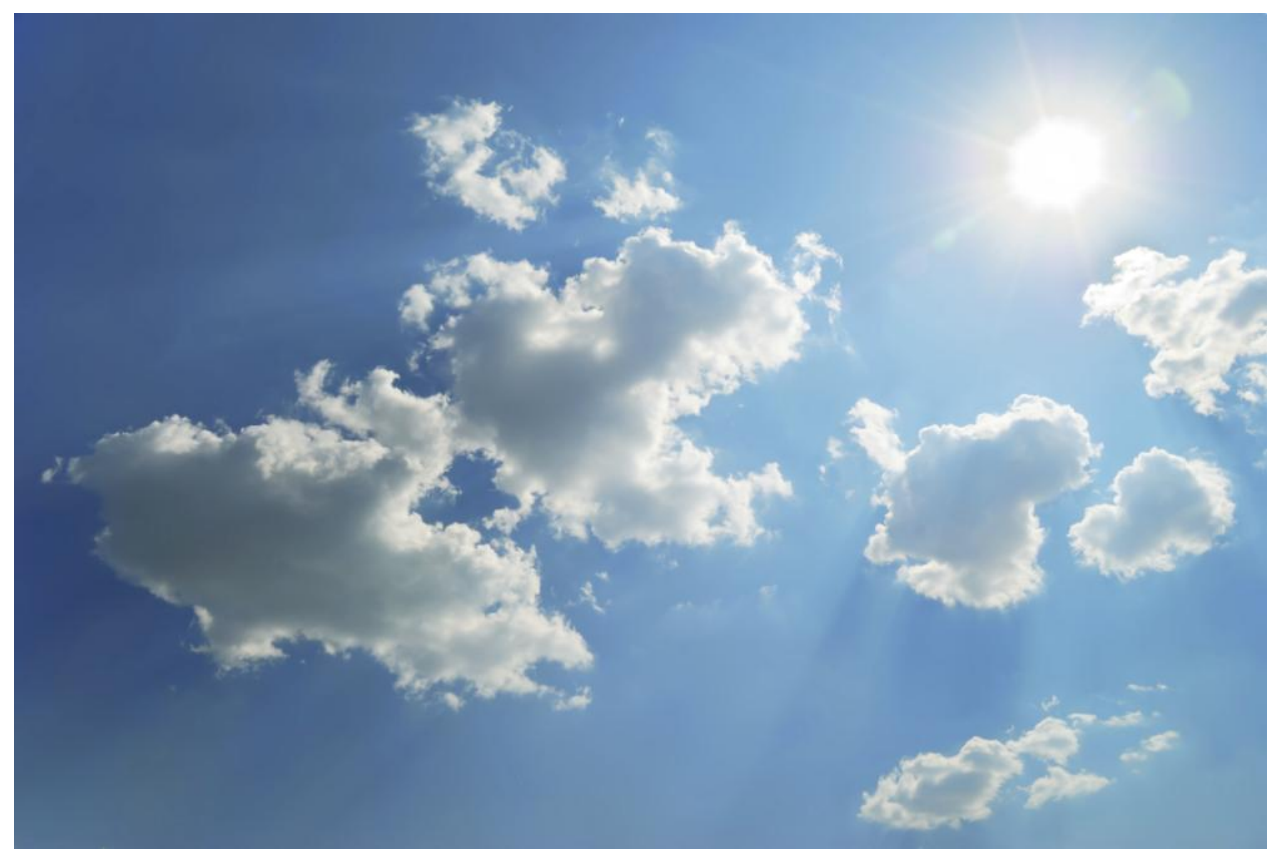

By Sue Ellen Hansen, Jeremy Iverson, Uwe Jensen, Hilde Orten, and Johanna Vompras

\section{DDI Working Paper Series - Longitudinal Best Practice, No. 2}

This paper is part of a series that focuses on DDI usage and how the metadata specification should be applied in a variety of settings by a variety of organizations and individuals. Support for this working paper series was provided by the authors' home institutions; by GESIS - Leibniz Institute for the Social Sciences; by Schloss Dagstuhl - Leibniz Center for Informatics; and by the DDI Alliance. 


\section{Enabling Longitudinal Data Comparison Using DDI EXPLOITING DDI'S FEATURES RELATED TO COMPARISON}

\section{PROBLEM STATEMENT/DESCRIPTION}

By their nature, longitudinal studies contain data from multiple points in time that can be usefully compared. Often, longitudinal studies also collect comparable data from various geographical regions, population samples, and data sources. For example, following are types of data collection done for some widely used longitudinal surveys:

- The European Social Survey (ESS), which monitors changing social attitudes and values across nations over time, is a repeated cross-section in-person survey conducted in 30 nations.

- The International Social Survey Programme (ISSP) has collected data annually since 1984 from crosssections of populations in 47 countries (as of 2010). ISSP collects common and country-specific data, and periodically field topical modules (such as "role of government") to gauge trends in attitudes (see Jensen et al., 2009).

- The U.S. Health and Retirement Study (HRS) collects data biennially from panel respondents nearing, at, or beyond retirement age, periodically adding new age cohorts. Data are generally collected in-person about living and deceased panel members, and may include physical measures, blood and saliva, and information from paper self-administered questionnaires. HRS also requests permission to collect data from administrative databases. Between waves HRS conducts additional surveys of selected sample respondents on focused topics.

- The Study of Health, Aging and Retirement in Europe (SHARE) has collected similar data in a series of rounds from 14 countries in Europe.

Several forms of documentation are useful for helping data users understand the relationships among waves of longitudinal data. These include cross-reference or concordance tables that make it easy to find the variables that hold a piece of information for different waves of data collection. For example, the SHARE Web site has concordance tables that display deviations in questionnaire items between waves and within waves across countries (http://www.share-project.org/; see also Kramer et al. on presenting longitudinal information to end users).

The DDI 3 life cycle model (Figure 1) is ideally suited to storing structured metadata that can be used as the source for these types of documentation. By thoroughly documenting the full process of the data life cycle, data producers can ensure that the similarities in their data are discoverable and usable by data producers, archives, and the research community. Using the standard's explicit comparison features, data producers can add an even richer description of the comparability of their data over time and other dimensions. Researchers can also use this explicit comparison model to describe similarities and differences among their data and other organizations' data.

The DDI 3 standard (hereafter referred to as DDI) allows data producers to provide enough transparency to give data analysts the tools they need to make informed decisions regarding comparative data analysis. 


\section{APPROACH}

For this paper, we took the approach of further defining and documenting the use of all of the DDI components related explicitly to comparison and harmonization in the context of the data life cycle. This seemed important because understanding how data items compare is essential to fully exploiting complex longitudinal data.

We started with basic ideas about comparing questions and variables and then moved on to describe the use of groups, subgroups, resource packages, and the DDI comparison module'. We also decided it would be useful to expand upon the DDI decision tree (see DDI Overview, Part I) with some added examples.

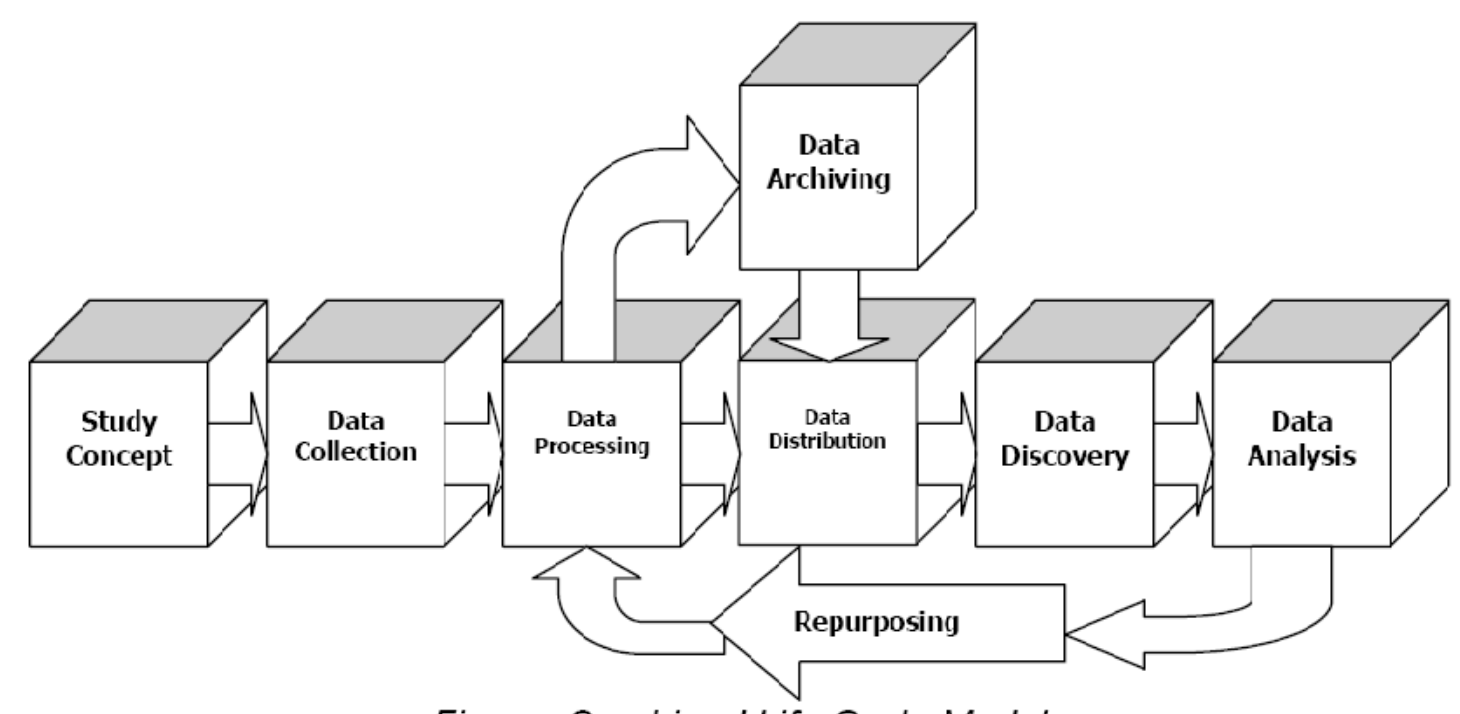

Figure: Combined Life Cycle Model

Figure 1: Data life cycle model

\section{Using DDI to Describe the Life Cycle}

The key to ensuring that relationships among items are well-documented is to use a consistent set of resources starting at the beginning of the study life cycle. To generate useful forms of documentation such as those described above, common metadata elements should be used.

At the beginning of a social research study, before any data exist or any questions are asked of respondents, researchers have in mind some things they want to study. These abstract ideas can be described in DDI as concepts. During the course of a study the research organization will write questions, collect data from many sources, and process and distribute the data, but all of these activities relate back to the original concepts on which the study is based.

1 The comparison module is the XML schema file comparative.xsd that contains the XML Schema type ComparisonType. 


\section{Implicit Data Comparison}

DDI allows researchers to link individual questions to the corresponding concepts that they attempt to measure. These questions are used to build a data collection instrument that could take the form of a telephone interview, a mail survey, or a survey in some other mode. Data are gathered during a period of data collection that uses one or more of these data collection instruments.

Similarly, after the data are collected data producers can describe the processes used to create public-use datasets from the collected data. Many types of processing activities can be documented: qualitative data coding, recoding from one code set to another, derivation and computation of variables, cleaning operations, and more.

Widely reusable elements may be described inside a ResourcePackage. Items inside a ResourcePackage can be referenced by one or more studies, or one or more waves or rounds of studies, and may contain references to other items.

The following table provides a summary of how DDI elements correspond to real world ideas. Each of the DDI constructs (except StudyUnit) can be stored in a ResourcePackage.

\begin{tabular}{|ll|}
\hline Logical Idea & DDI Construct \\
Abstract ideas & Concept \\
Survey questions & Question \\
& Category \\
Survey instruments & Instrument \\
Data collection waves & CollectionEvent \\
& StudyUnit \\
Datasets & Physicallnstance \\
Columns of data & Variable \\
Response options & RepresentationType \\
(applicable for Questions and Variables) & \\
\hline
\end{tabular}

Table 1: Mapping survey components to DDI

DDI also has constructs that can be used to represent relationships among real world ideas. For example:

\begin{tabular}{|ll|}
\hline Logical Idea & DDI Construct \\
What Concept does a Question try to measure? & Question \\
$\begin{array}{l}\text { From what Question does a Variable get its data? } \\
\text { Do Questions change over time, space, mode, or }\end{array}$ & Variable \\
sample? & SourceQuestion \\
In what surveys does a Question appear? & StudyUnit \\
Are Questions replicated across waves? & DataCollection \\
& Instrument \\
When and how often was a survey administered? & DataCollection \\
& CollectionEvent \\
How are certain variables derived, created, or & StudyUnit \\
changed? & Generationlnstruction for all \\
How does raw data from a survey get turned into & ControlOperation or CleaningOperation as appropriate \\
public use data? & DataProcessingEvent \\
\hline
\end{tabular}

Table 2: Mapping survey relationships to DDI 
Metadata tools can analyze the structure of such life cycle metadata to determine common items and common relationships among items (study units, questions, variables, concepts, instruments, data collection events, etc.). By using tools in this manner, data producers do not need to create and manage redundant metadata explicitly specifying comparable items. This results in reduced effort spent and more reliable documentation for longitudinal studies.

\section{Explicit Datal Comparison}

Using DDI's groups, subgroups, and the comparison module, data producers can add an even richer description of the comparability of their data. The Group module allows comparison over the following basic dimensions (see the DDI Overview).

- $\quad$ Time

- Instrument

- Panel

- Geography

- Dataset

- Language translation

Furthermore, researchers can use the Comparison module to explicitly document similarities and differences in their data. This module can be applied according to the general strategy chosen to organize and reuse metadata -- the ResourcePackage and/or the Group module.

The module documents comparable items from two different schemes. Presently the module facilitates comparison mapping universes, concepts, questions, categories, coding schemes, and variables. The comparison is pairwise and unidirectional from a source to a target item. For example, a harmonized variable would be represented by the target item compared to the source item.

The relationship between the two items can be described for common and different aspects as textual descriptions and also as a formalized description using a controlled vocabulary. Additionally, the degree of commonality and the correspondence can be defined.

CodingScheme provides particular options for describing the derivation process transforming one coding scheme into another. Options include a human-readable description of the translation process (e.g., Source code 1 through 3 equal Target code 1), a command line for a specified command language, or the use of the GenerationInstruction from DataCollection. For example a direct mapping of each source code value to its target value could be declared. (See DDI_3.1_Part_II_UserGuide for examples and guidance to classify level of comparison at line $2344 \mathrm{ff}$.)

\section{Versioning}

In addition to using implicit comparison available by inspecting the life cycle metadata, and the explicit comparison available using DDl's comparison module, researchers may also gain insight into the comparability of items based on an item's version history.

In DDI, if an item is changed after it has been used it must be given a new version number. For example, if a question asked in one year has an additional response option the following year, the same question is used for both years but with a different version. 
By looking at the history of changes over time, a researcher can analyze what similarities or differences are applicable to her research. For example, to facilitate research ESS provides a Download Wizard for the cumulative data file that shows differences in variable names, questions, and response options across rounds (available at http://ess.nsd.uib.no/downloadwizard/). This file could be automatically generated by a tool if the different versions of the variables and questions are documented in DDI.

Versioning also facilitates describing how derived variables are produced (e.g., harmonization of versions of variables over time), and providing associated analysis command setup text (i.e., SAS or SPSS), either as inline information or as an external file. The derivation process can be formalised in an eXtensible Markup Language (XML) language, like MathML, independently from statistical packages, thus making commands for different target formats like Stata or SPSS possible (see Wackerow 2008).

\section{Additional Metadata thatt Enable Comparison}

Many other types of general study metadata that describe study processes are also useful for researchers to determine whether specific data should be compared. These include metadata about the following:

- Sample design

- Questionnaire design and development (e.g., focus groups, translation, adaptation, pretesting)

- Instrument technical design (e.g., formatting and/or programming an instrument, including testing)

- Interviewer recruitment, selection, and training

- Data collection

- Data processing and statistical adjustment

- Data harmonization and dissemination

- Process monitoring and quality control

DDI currently has some elements that may be used for capturing metadata on these processes, but more are needed. To meet this need, the DDI Alliance has established a working group on Survey Design and Implementation (SDI) that is developing proposals for extensions to DDI that would permit richer description of study metadata.

\section{Using DDI Resource Packages, Groups, Subgroups, and Comparison} Modules

DDI resource packages, groups, subgroups, and comparison modules can enable comparison and harmonization of data for various study units (for example waves, instruments, studies) across the dimensions listed above and as shown in the figure below. 


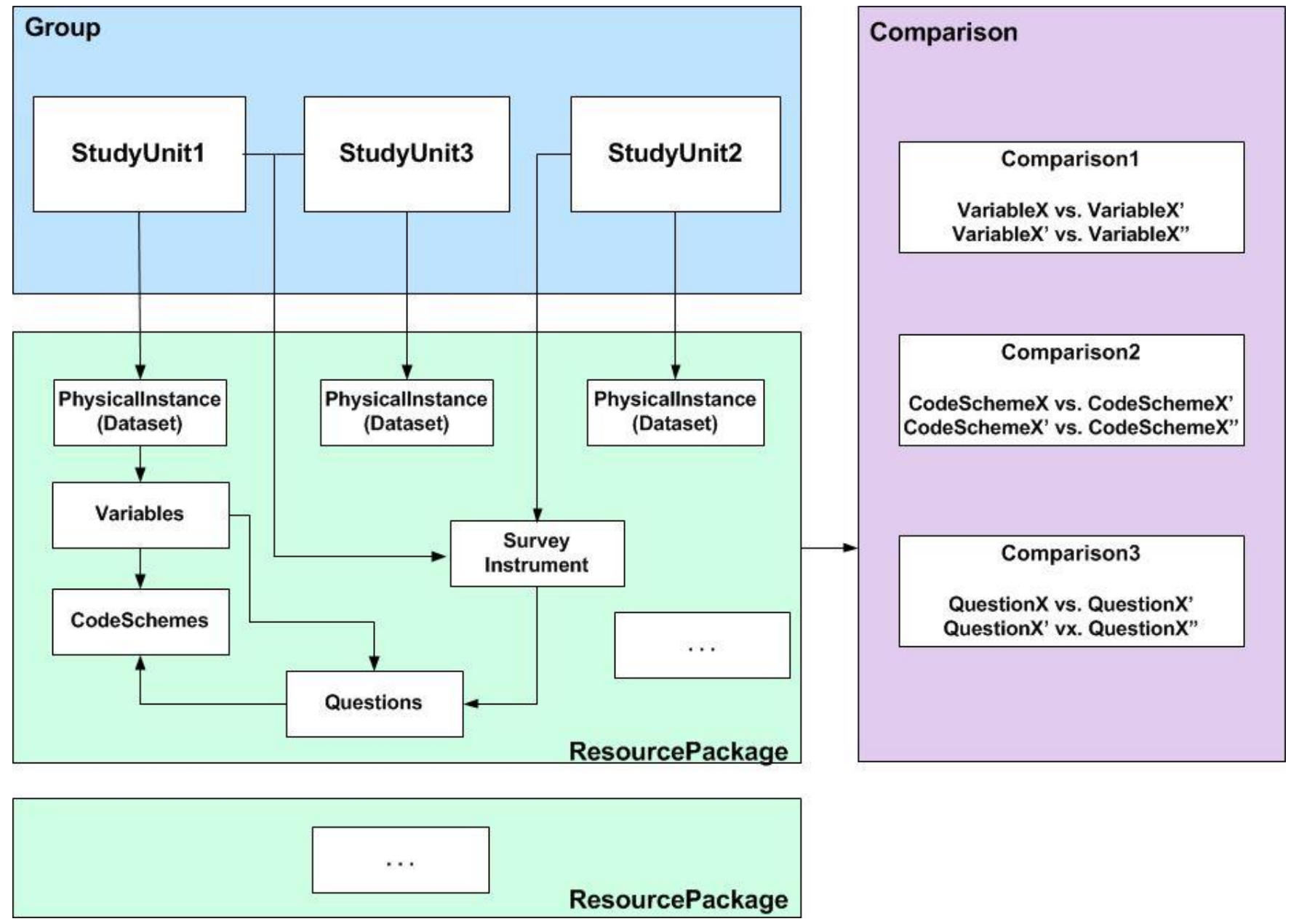

Figure 2: Using Resource Packages, Groups, Subgroups, and Comparison Modules

Groups may be used to make pair-wise comparisons between subgroups and study units using the comparison module, and subgroups may be used in a similar fashion to make comparisons between study units. Resource packages provide metadata that may be reused across groups, subgroups, and study units.

\section{Expanded Decision Trees for Documenting Comparison}

Following are decision trees that may be used to determine what group attributes to use to document and compare types of longitudinal studies. Attributes not relevant to longitudinal surveys are not shown, e.g., "PO" for a non-panel survey. 


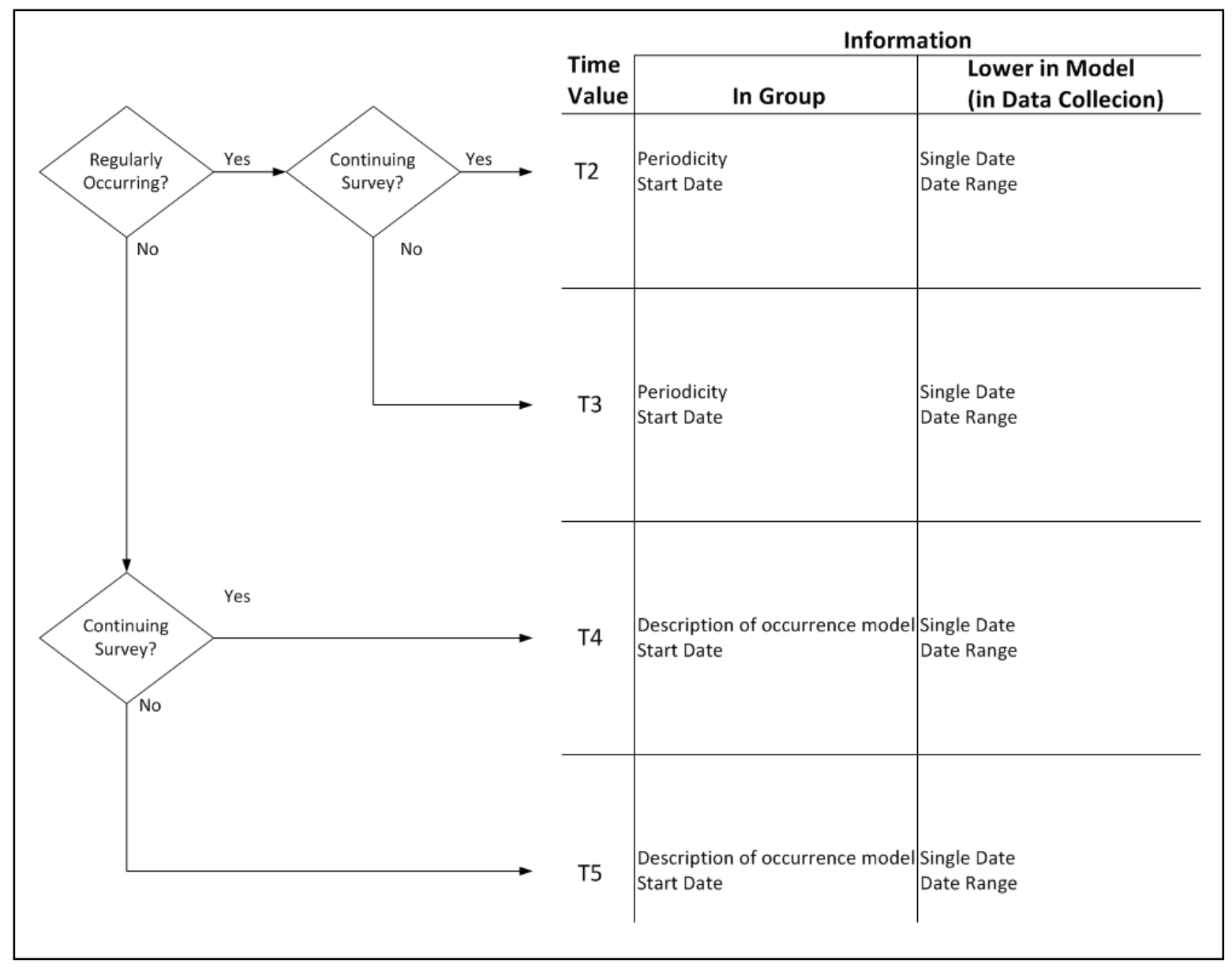

Figure 3: Decision Tree to document and compare Time 


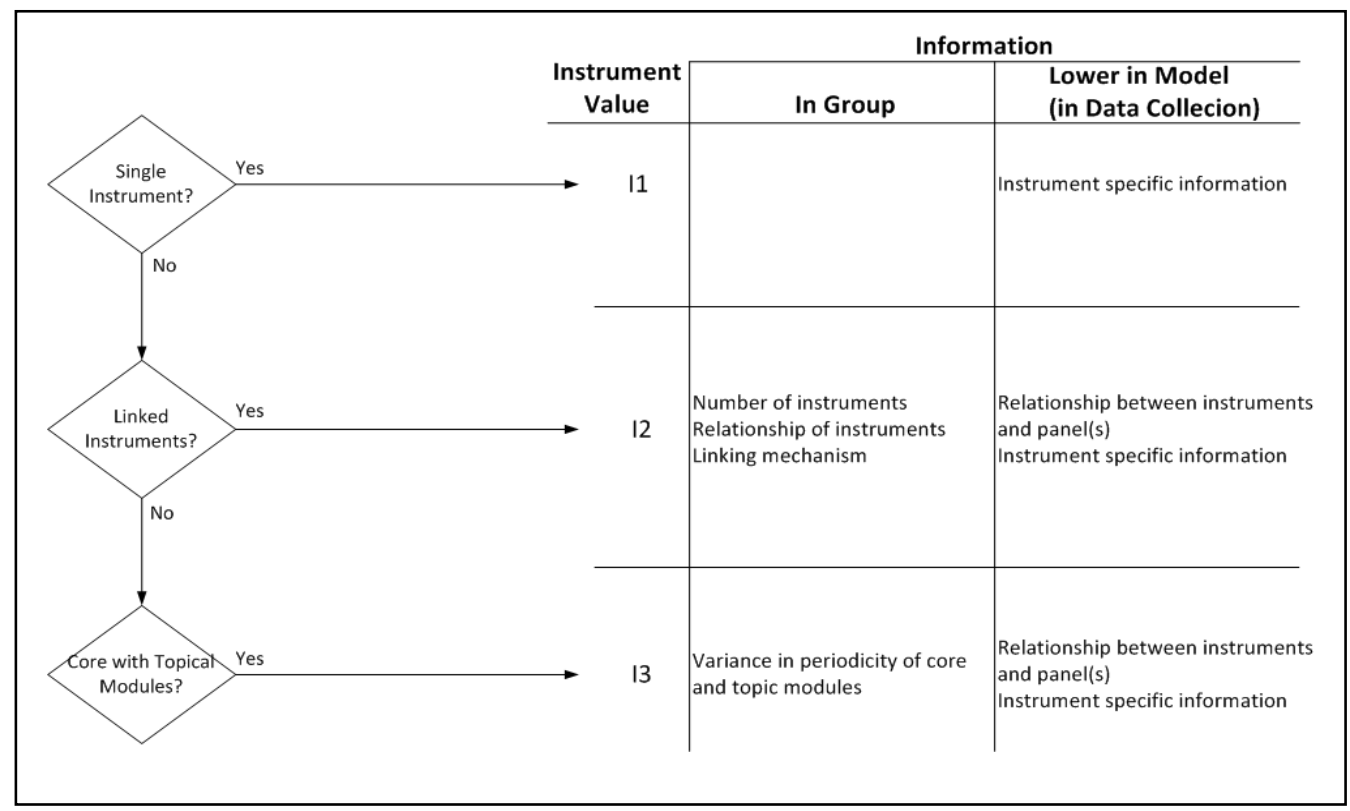

Figure 4: Decision Tree to document and compare Instrument

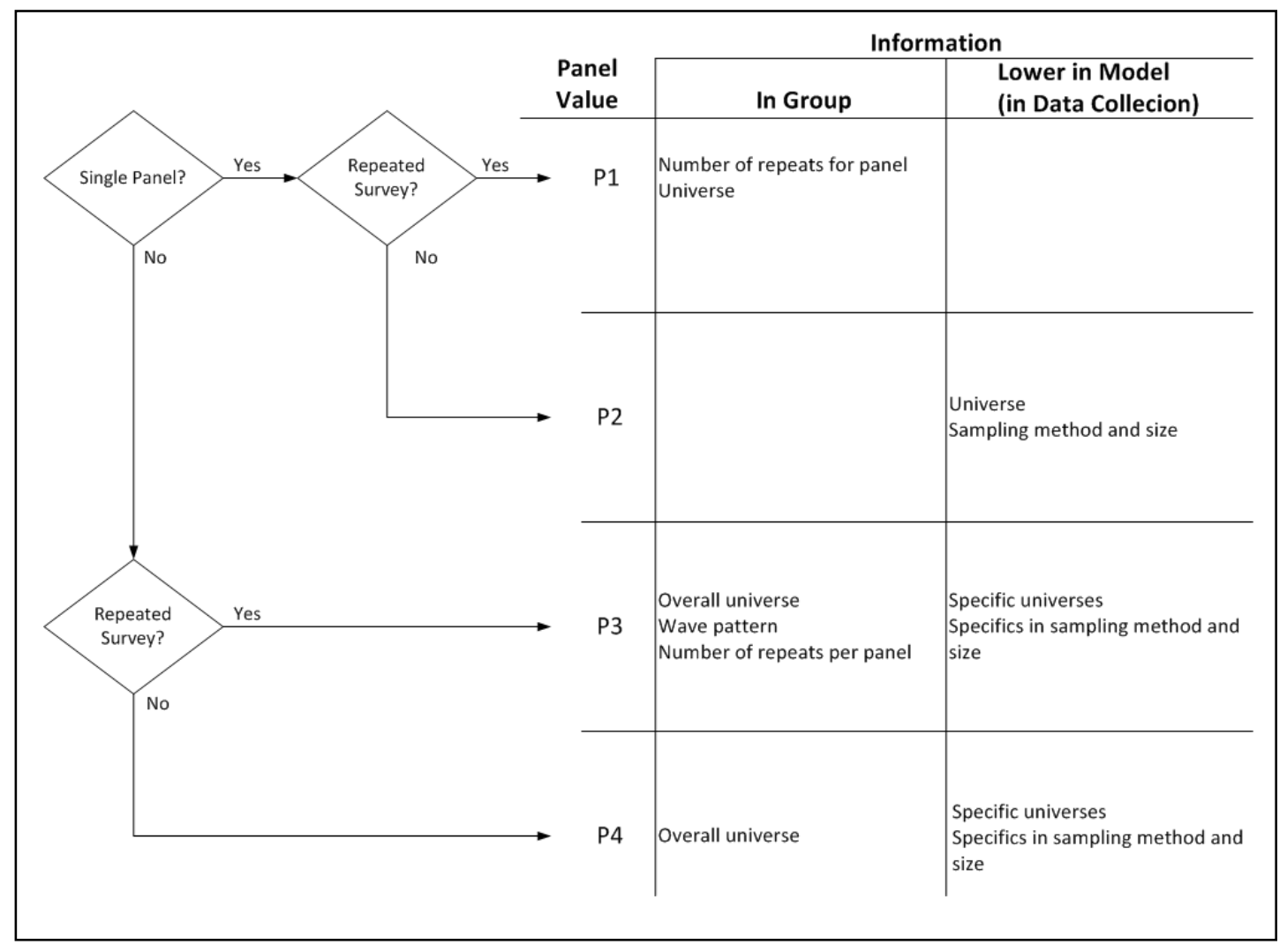

Figure 5: Decision Tree to document and compare Panel Data 


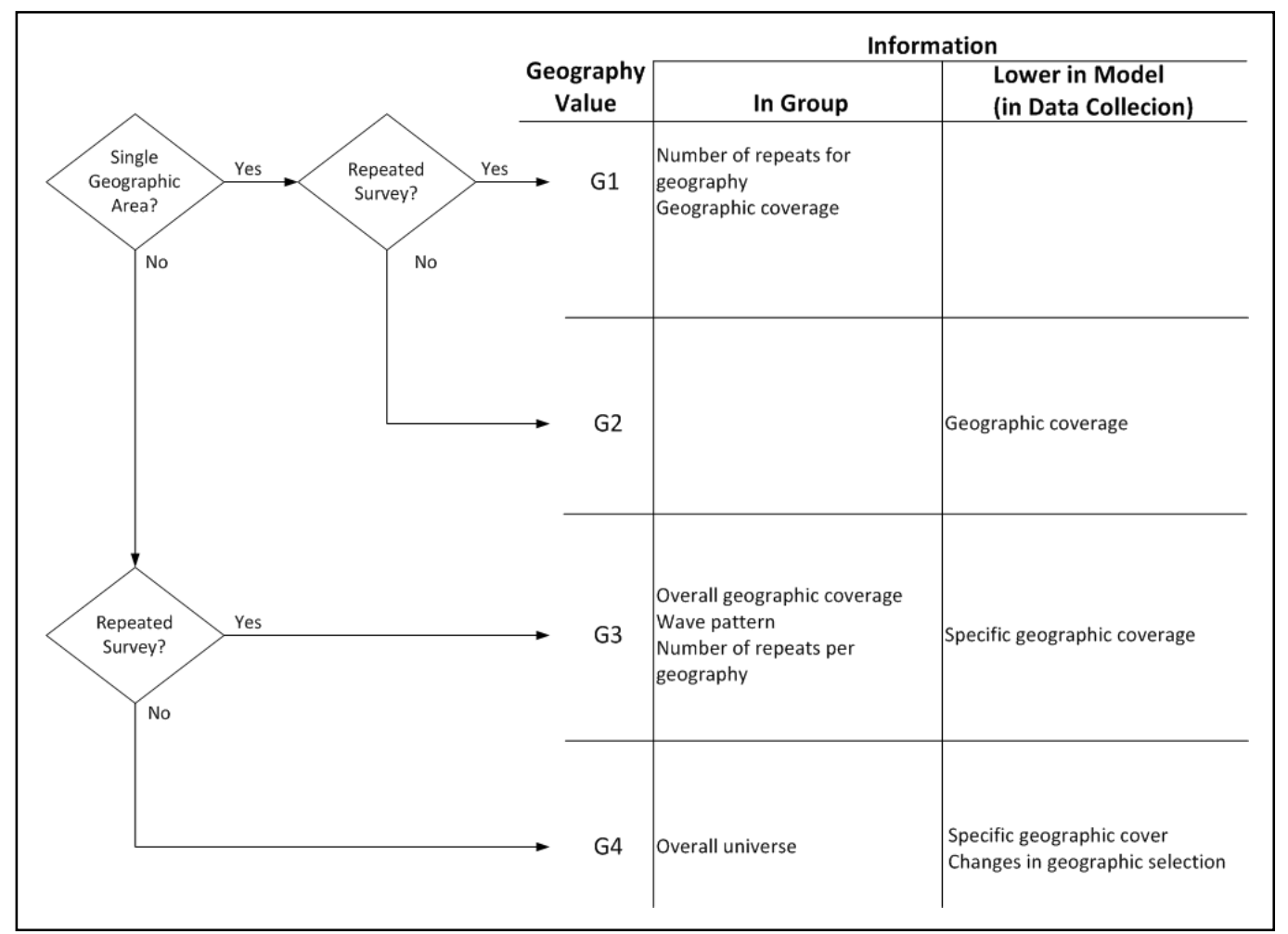

Figure 6: Decision Tree to document and compare Geography

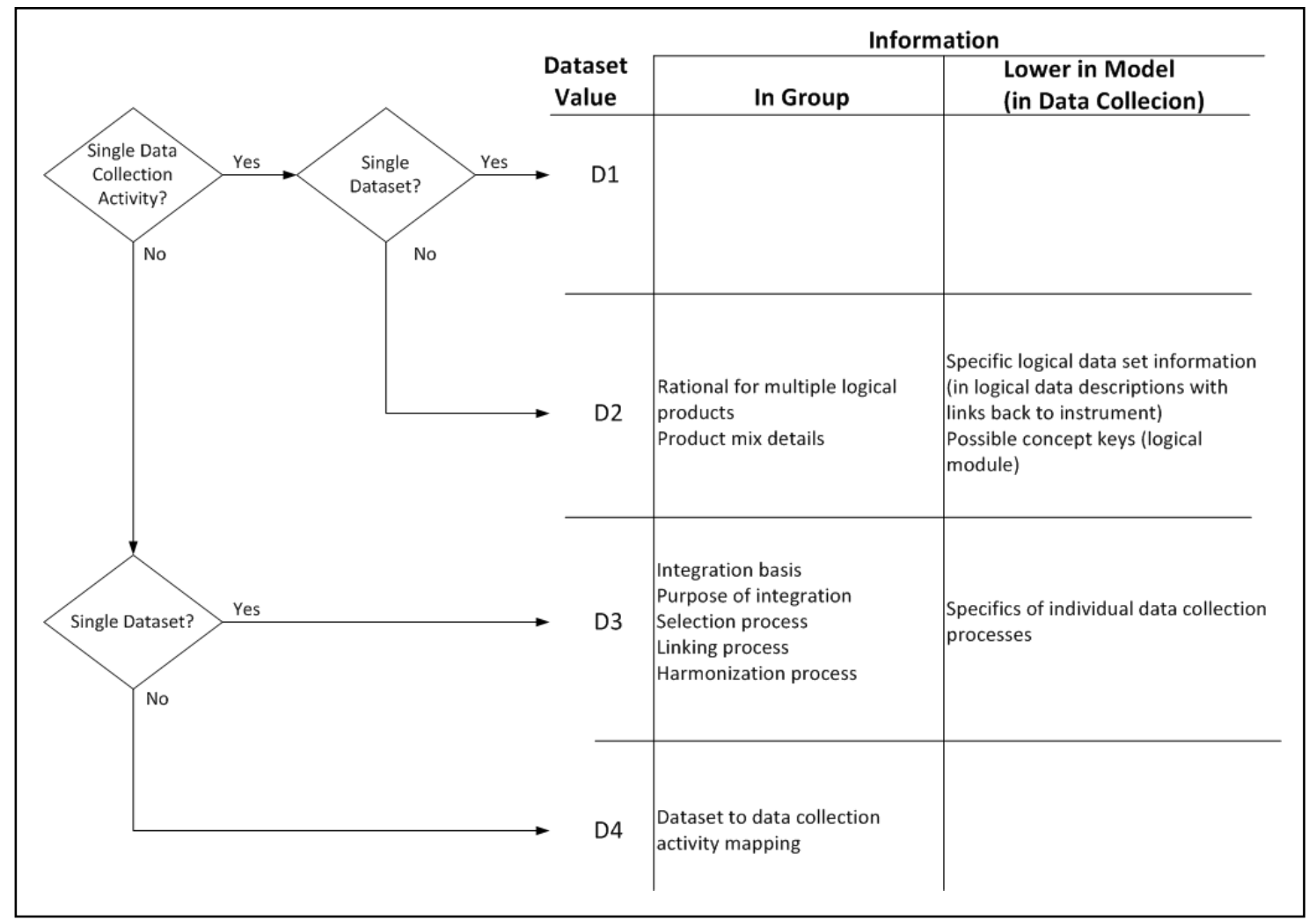

Figure 7: Decision Tree to document and compare Datasets 


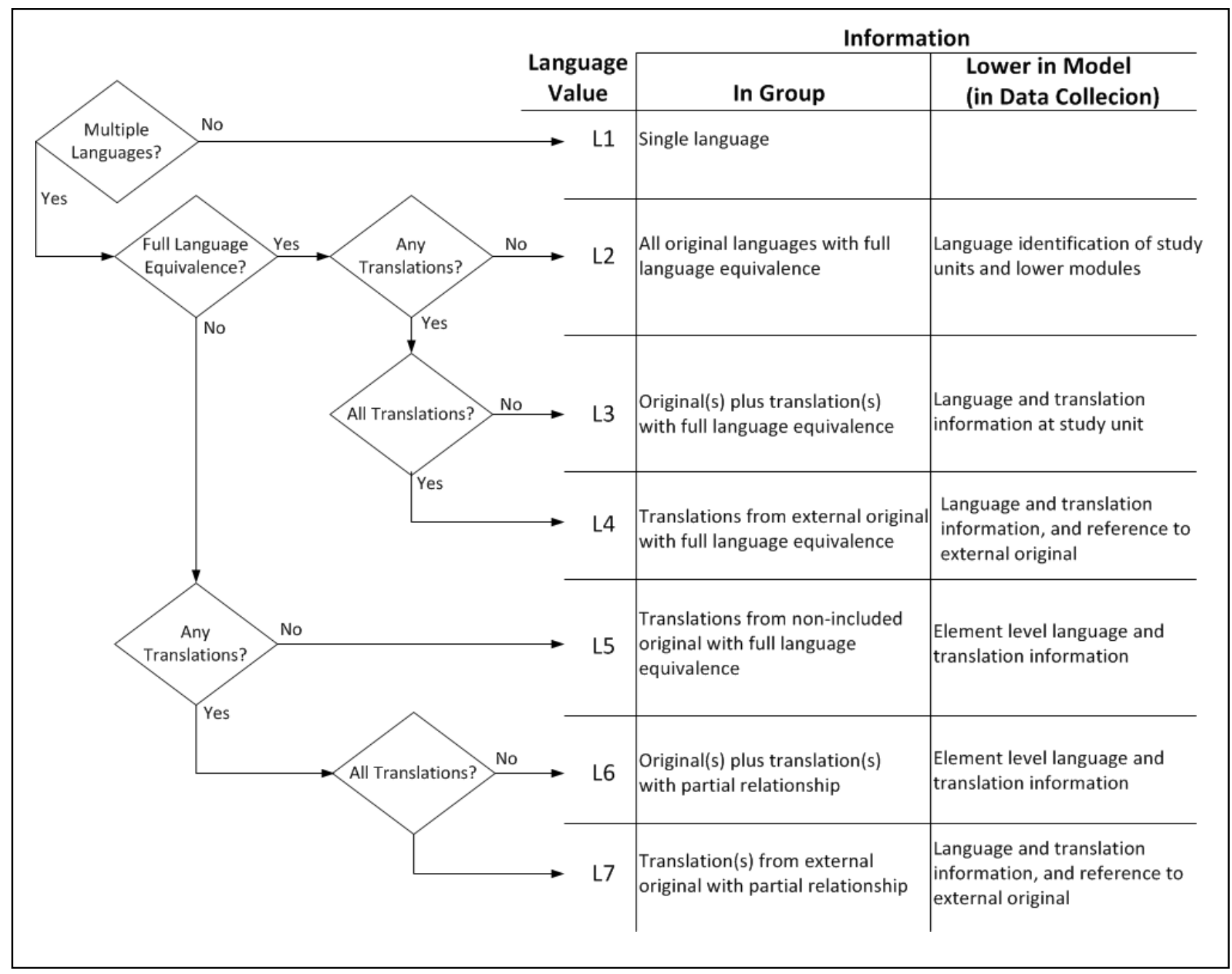

Figure 8: Decision Tree to document and compare Language

\section{Use Cases}

Following are three examples of research questions related to comparison and harmonization of data that may be addressed through the use of well-documented survey metadata produced through the use of the DDI standard. To create such documentation, data producers use DDI-compliant tools to extract instrument and question-level XML metadata from information provided via data collection software. They may also use tools to create study level XML metadata (such as for sampling, translation, and interviewer training). Data producers and archivists then may use tools that utilize the constructs described above for evaluation of available metadata for comparison and documentation of similarities and differences in data across studies, time, geography, language translations, etc.

\section{Example 1}

A researcher wishes to study education over time. She downloads a cumulative dataset containing data for several rounds of a repeated cross-section survey. The researcher wants to discover if the education question in the cumulative dataset is identical over all rounds, and if not, how many versions of the variable there were. 
If the education questions are not identical, the researcher would like to explore whether the different versions could be harmonized. On their Web site, the data producers provided metadata about question versions across rounds that allowed her to answer these questions. They were able to do this using a resource package with education variable-level information used by all grouped study unit (round) instruments and the comparison module to compare questions (modeled in Figure 9). The researcher learned that the education questions had not changed over time and that the education data were comparable.

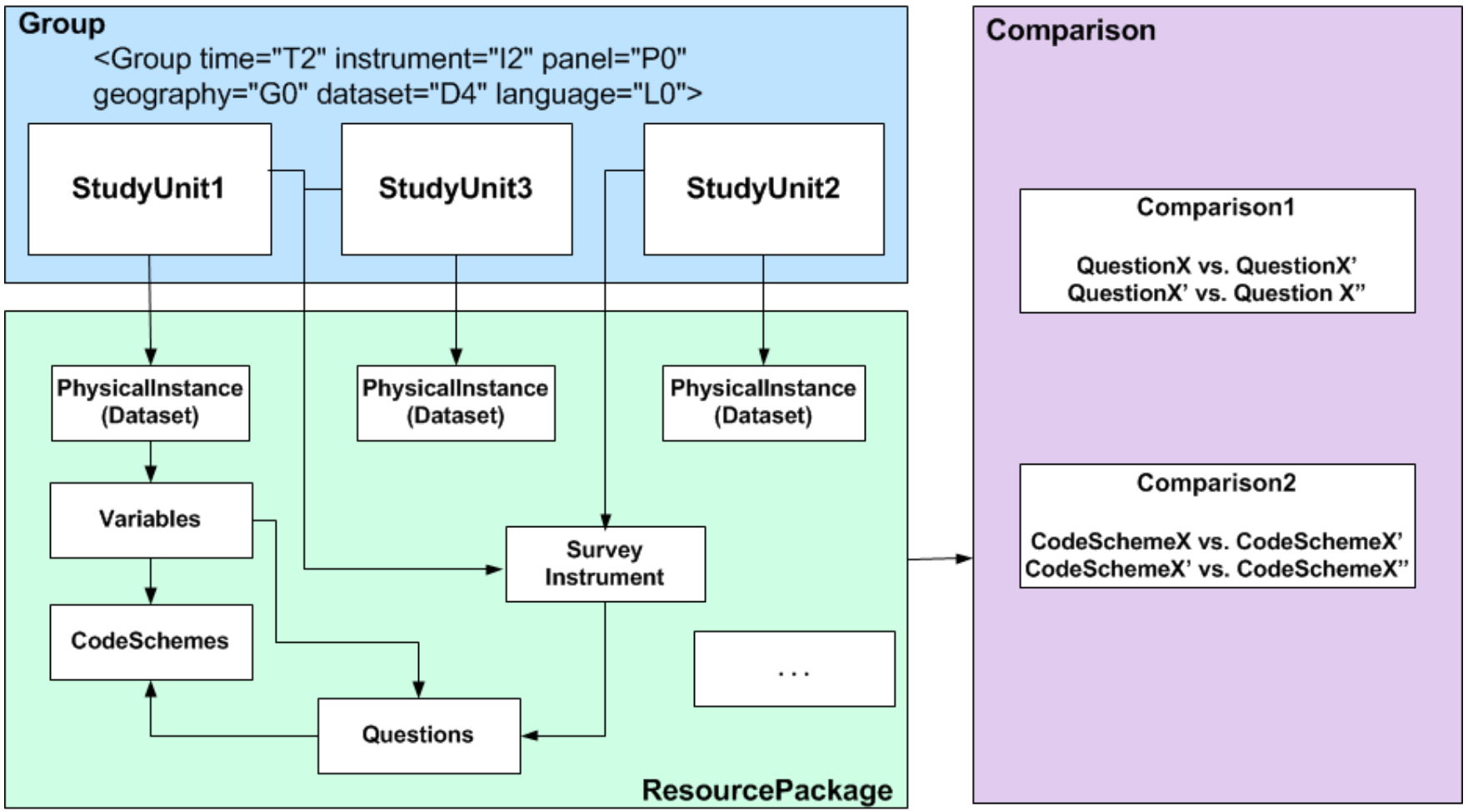

Figure 9: Using ResourcePackage for variable information for linked instruments and the comparison module to compare questions and response options

\section{Example 2}

A researcher is analyzing data across waves of a panel study. She wants to know if there has been a refreshment of the sample in any of the waves, and if so, what the sampling procedures were in each wave. On their Web site, the data producers provided metadata about sample design and sampling procedures across rounds of data collection. They were able to do this using a resource package with data collection methodology information used by all grouped study unit (panel round) data collection events and the comparison module to compare sampling procedures used (modeled in Figure 10). With the information provided, she was able to determine that the sample was refreshed in the third round, that the sampling procedures had changed, and why they had changed. 


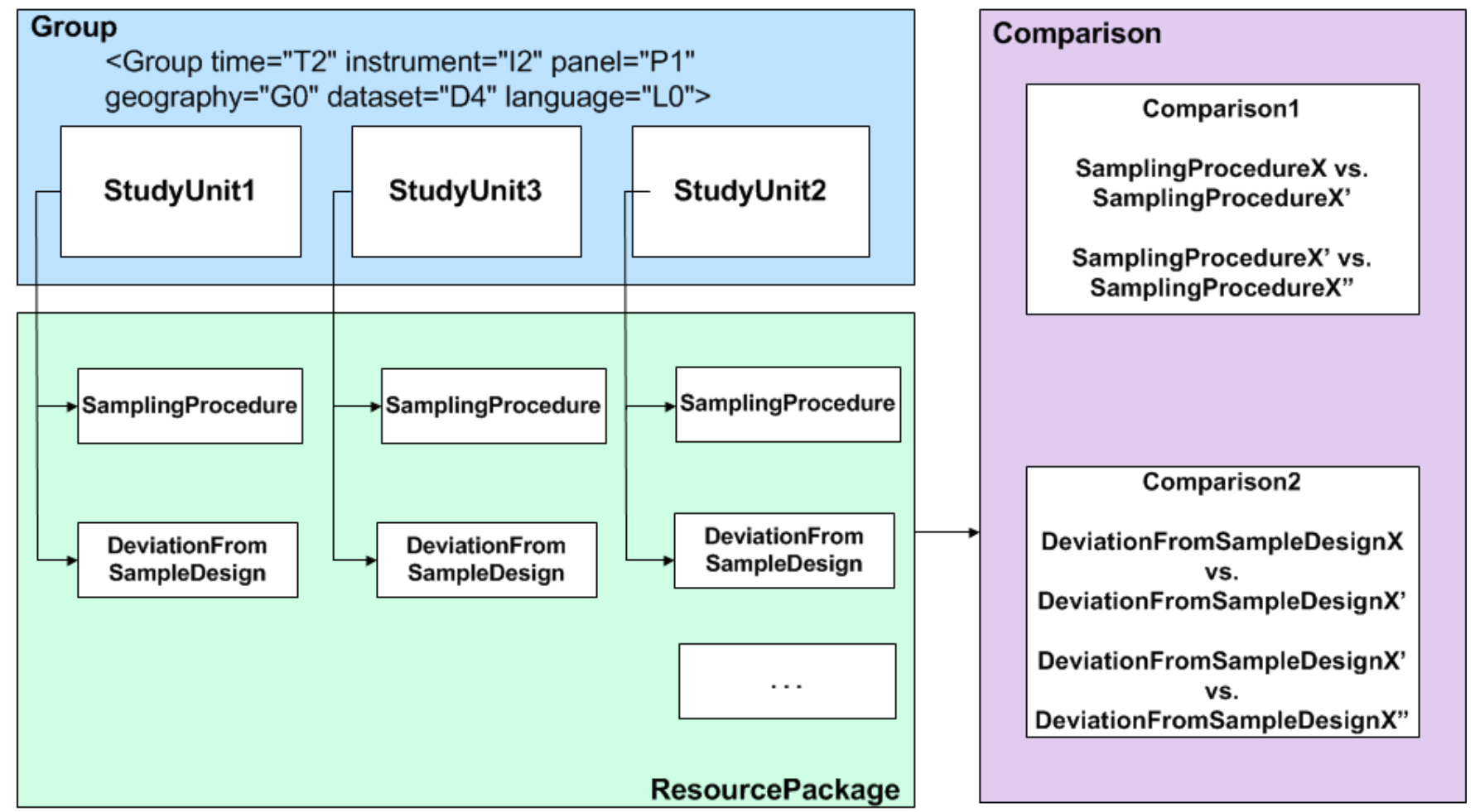

Figure 10: Using ResourcePackage for data collection methodology information for study waves and the comparison module to compare sampling procedures

\section{Example 3}

A researcher is designing a new, international study. The study will be based on an existing survey that has proved useful in other locations. Naturally, the researcher would like to reuse as much documentation as possible from the previous study.

Some of the variables in the existing study have several different versions, and the researcher would like to compare these before deciding which to use. The researcher would also like to compare the translation of questions into particular languages, since these sometimes vary among countries fielding the same survey in different languages.

Using the documentation on the study Web site, the researcher is able to view translations of question and response option text between the source instrument and country translations, as well as differences across versions of variables in each instrument. 


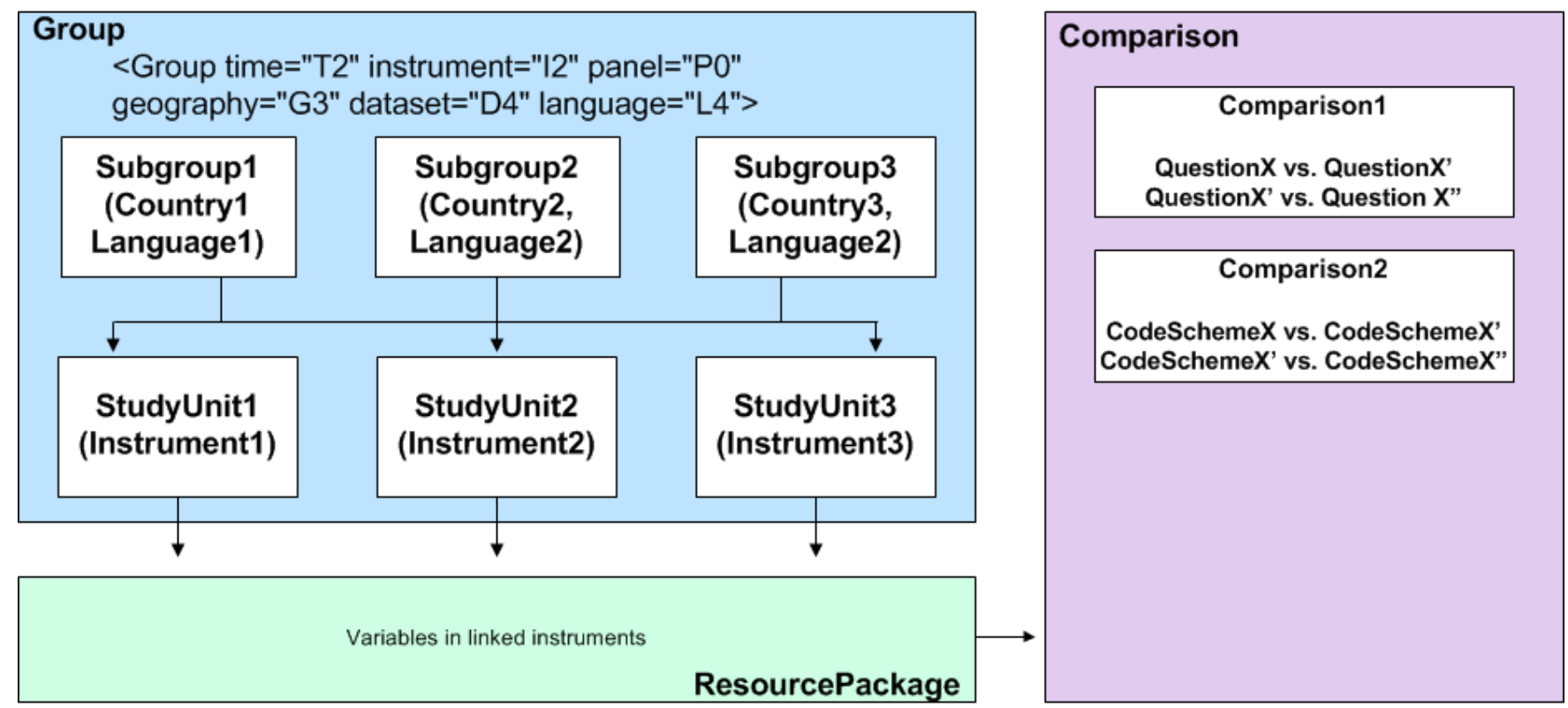

Figure 11: Using ResourcePackage for variables, and the comparison module at the subgroup and study unit levels for comparison of variable versions and translations 


\section{RECOMMENDATIONS}

Currently, the DDI 3.1 comparison module allows pairwise comparison of two items. For two given items, the following metadata may be specified.

\begin{tabular}{ll}
\hline Commonality & $\begin{array}{l}\text { Human-readable text describing the commonality between } \\
\text { the two items }\end{array}$ \\
Difference & $\begin{array}{l}\text { Human-readable text describing the differences between } \\
\text { the two items }\end{array}$ \\
Commonality Type & $\begin{array}{l}\text { A string defining the type of comparison. User-defined, but } \\
\text { hopefully using a well-known or controlled vocabulary for } \\
\text { machine-actionability }\end{array}$ \\
\hline
\end{tabular}

While this sort of comparison is useful, it would also be useful to compare many items at once instead of two at a time, pairwise. Currently, in order to non-ambiguously document the comparability of several items, each combination must be documented independently. For example, if a researcher wants to document the similarities among 20 concepts, she will need to define 190 correspondences in DDI 3.1, repeating the text in the commonality and difference fields each time.

Expanding the types of items that can be compared would also be useful. DDI 3.1 allows comparison of concepts, variables, questions, categories, codes, and universes. Other useful types of items that might be compared include Instruments and Control Constructs (used to document the logic of the flow of questions in an instrument).

Note that we do not recommend using group inheritance as a comparison method since inherited items cannot be referenced by other organizations. In addition, the meaning of the group @action attribute (add, update, delete) is ambiguous. It impossible to tell in which way the producer intended the inheritance to work, which depends on whether the original DDI specification or the corrigendum-updated specification was used (see Corrigendum to Technical Specification, DDI 3.1).

\section{REFERENCES}

Uwe Jensen with Sanda lonescu, Mari Kleemola, Agostina Martinez, Wendy Thomas, Mary Vardigan, and Wolfgang Zenk-Möltgen. "Grouping of Survey Series Using DDI 3." DDI Working Paper Series - Use Cases, No. 6, April 2010. http://dx.doi.org/10.3886/DDIUseCases06

Ionescu, Sanda, with Larry Hoyle, Mari Kleemola, Martin Mechtel, Olof Olsson, and Wendy Thomas. "Using DDI 3 for Comparison." DDI Working Paper Series - Use Cases, No. 3, April 2010. http://dx.doi.org/10.3886/DDIUseCases03

Hoyle, Larry, Castillo Fortunato, Benjamin Clar, Neeraj Kumar Kashyap, Denise Perpich, Joachim Wackerow, and Knut Wenzig. "Metadata for the Longitudinal Data Life Cycle: The Role and Benefit 
of Metadata Management and Reuse." DDI Working Paper Series, Longitudinal Data Best Practices, Number 3, December 2010. http://dx.doi.org/10.3886/DDILongitudinal03

Kramer, Stefan, Randy Banks, Vicky Chang, Mary Vardigan, and Wolfgang Zenk-Möltgen. "Presenting Longtiudinal Studies to End Useres Effectively Using DDI Metadata." DDI Working Paper Series, Longitudinal Data Best Practices, Number 4, December 2010. http://dx.doi.org/10.3886/DDILongitudinal04

Joachim Wackerow. "DDI Perspective on Harmonization." CESSDA PPP Expert Workshop on Harmonization Issues in Comparative Surveys. Paris, April 2008.

Data Documentation Initiative. DDI 3.1 Schema and Documentation - Part I - Overview, Schema and Field Level Documentation. http://www.ddialliance.org/specification/ddi3.1\#3.1 schema

Corrigendum to Technical Specification, DDI 3.1. http://www.ddialliance.org/Specification/DDILifecycle/3.1/XMLSchema/Documentation/Corrigendum 10-01-2010.pdf. 


\section{APPENDIX A}

\section{GLOSSARY}

Administrative data

Biomarker

Cohort/Event-based

\section{Concordance}

Continuous panel

Continuous time series

Cross-sectional

Cross-sectional ad-hoc followup
Data collected for the administration of government (or other) programs. Examples include:

- Economic data

- Educational achievement in public schools

- Hospital admissions/discharges/outcomes

- Income/sales/property tax records (both personal and business)

- Immigration applications/approvals/naturalization records

- Social Security records

- Unemployment Insurance claims/records

- Voting records

- Workers compensation (for on-the-job injuries)

The official NIH definition of a biomarker is: "a characteristic that is objectively measured and evaluated as an indicator of normal biologic processes, pathogenic processes, or pharmacologic responses to a therapeutic intervention."

Ref: Biomarkers Definitions Working Group: "Biomarkers and Surrogate Endpoints: Preferred Definitions and Conceptual Framework." CLIN PHARMACOL THER 2001;69:89-95.

\section{http://www.everythingbio.com/glos/definition.php?ID=3716}

Data collected over time about a group of individuals that are connected in some way or have shared some significant experience within a given period. Examples: birth, disease, education, employment, family formation, participation in an event.

Tool or table indicating the presence of the same variable or question over waves of a study.

Reports from a panel collected on a regular basis.

Phenomena measured at every instant of time. Examples: lie detectors, electrocardiograms, etc.

Data about a population obtained only once.

Data collected at one point in time to complete information collected in a previous cross-sectional study; the decision to collect follow-up data is not included in the study design. 


\section{Data harmonization}

Data life cycle

DDI

Digital Object Identifier (DOI)

Discrete time series

DSS / HDSS

Grouping
Data harmonization is the process of bringing variable-level information into alignment to express comparability. This is often done through mapping across various elements of the variables, including variable name, label, categories, codes, etc.

The whole course of existence of a set of data, from initial conception to ultimate disposal.

The Data Documentation Initiative (http://www.ddialliance.org/). Also that organization's metadata specification for the social and behavioral sciences.

A character string used to uniquely identify an electronic document or other object. Metadata about the object is stored in association with the DOI name and this metadata may include a location, such as a URL, where the object can be found. The DOI for a document or dataset is permanent, whereas its location and other metadata may change. Referring to an online document by its DOI provides more stable linking than simply referring to it by its URL, because if its URL changes, the publisher need only update the metadata for the DOI to link to the new URL.

Measurements taken at (usually regularly) spaced intervals.

Health and Demographic Surveillance Systems (HDSS) for longitudinal monitoring of small-area populations by continuous recording of vital events have been set up in many developing countries. HDSS's are based on a data gathering method comprising an initial census of the resident population, followed by multi-round surveys covering all inhabitants of the area. They thus, provide a geographical and temporal observation window on a locally circumscribed population defined using certain rules of residence. Individuals' life events during their period(s) of residence in the survey area are recorded on an individual basis (the minimum data being births, deaths and migration), but sometimes per household or per residential unit. Examples: macroeconomics (weekly share prices, monthly profits, sales); meteorology (daily rainfall, hourly temperature); measurements of individuals (blood pressure, weight, height); sociology (crime figures, employment figures), etc.

A DDI mechanism to clearly document the repurposing of aspects of the initial study and the relationships that exists between each of the component studies in the group. The typical use case involves a series or collection of studies which are related in some way or a group of studies which are being compared. A Group can be comprised of StudyUnits and SubGroups. A standard set of attributes describes the following dimensions for grouping: Time, Instrument, Panel, Geography, Datasets, Language. 
Instrument

Interval panel

Longitudinal

Panel

Published

Register data

Resource package

Retrospective study

Surveillance study

Time series

Trend/Repeated cross-section

Trials / Interventions

Versioned

Wave
A specific instrument or tool used to collect data. For survey data, the instrument has traditionally been seen as a questionnaire, but devices used to collect biomedical information, e.g., fMRI scanning devices, can also be viewed as instruments.

Measurements taken only when information is needed.

Data collected repeatedly over time to study change in a population.

Data collected over time from, or about, the same sample of respondents.

The DDI attribute isPublished is set to true when the metadata are made available outside of the group of original developers. Published metadata must be versioned.

Data collected and maintained on individuals and businesses to track vital statistics and other information.

A means of packaging any maintainable set of DDI metadata for referencing as part of a study unit or group. A resource package structures materials for publication that are intended to be reused by multiple studies, projects, or communities of users. A resource package uses the group module with an alternative top-level element called Resource Package that is used to describe maintainable modules or schemes that may be used by multiple study units outside of a group.

A study in which data are collected from recollections of past events.

A study in which data are collected by systematic observation.

Data collected repeatedly over time to study change in observations. These are typically "objective" measurements of phenomena that can be observed externally, as opposed to attitudes/opinions or feelings. Examples may include economic/financial indicators, natural/ meteorological phenomena, vital statistics, etc.

The study of different samples/different groups of people from the same population at several points in time, using the same set of questions/variables. Conclusions are drawn for the population. Examples: public opinion polls, elections studies, etc.

A study involving some sort of experimental action usually in comparison to some control condition.

Metadata for which any changes will require an update of the version attribute of the metadata.

One of a sequence of repeated stages of a study. 


\section{APPENDIX B}

\section{ACKNOWLEDGMENTS}

The paper is one of several papers that are the outcome of a workshop held at Schloss Dagstuhl - Leibniz Center for Informatics in Wadern, Germany, on October 18-22, 2010. The series was edited by Stefan Kramer, Larry Hoyle, and Mary Vardigan.

\section{Workshop Title:}

The Data Documentation Initiative (DDI) Standard : Managing Metadata for Longitudinal Data - Best Practices

Link: http://www.dagstuhl.de/10422

\section{Organizers:}

Arofan Gregory (Open Data Foundation, Tucson, Arizona, USA)

Mary Vardigan (Inter-university Consortium for Political and Social Research [ICPSR], University of Michigan, USA) Joachim Wackerow (GESIS, Leibniz Institute for the Social Sciences, Germany)

Participants in the workshop:

- Christian Bilde Andersen, Danish Data Archive (DDA)

- Randy Banks, Institute for Social and Economic Research (ISER), University of Essex

- Bill Block, Cornell Institute for Social and Economic Research (CISER), Cornell University

- Daniel Bontempo, Life Span Institute, University of Kansas

- Fortunato Castillo, MRC Centre of Epidemiology for Child Health, Institute of Child Health, University College London

- Vicky (Huey-Chi) Chang, Wisconsin Longitudinal Study, University of Wisconsin-Madison

- Benjamin Clark, London School of Hygiene and Tropical Medicine, Tazama Project, Tanzania

- Sue Ellen Hansen, Institute for Social Research, Survey Research Operations, University of Michigan

- Stan Howald, Wisconsin Longitudinal Study, University of Wisconsin-Madison

- Larry Hoyle, Institute for Policy and Social Research, University of Kansas

- Jeremy Iverson, Algenta Technologies

- Uwe Jensen, GESIS - Leibniz Institute for the Social Sciences

- Douglas Kieweg, Center for Biobehavioral Neurosciences in Communication Disorders (BNCD), University of Kansas

- Neeraj Kumar Kashyap, Vadu Rural Health Program, KEM Hospital Research Centre, INDEPTH Network

- Stefan Kramer, Cornell Institute for Social and Economic Research (CISER), Cornell University

- Hilde Orten, Norwegian Social Science Data Archive (NSD)

- Denise Perpich, Language Acquisition Studies Lab, University of Kansas

- Barry Radler, Institute on Aging, University of Wisconsin-Madison

- Ingo Sieber, German Institute for Economic Research (DIW) Berlin, Socio-Economic Panel Study (SOEP)

- Johanna Vompras, University Bielefeld Library, Germany

- Knut Wenzig, National Educational Panel Study (NEPS), University of Bamberg

- Wolfgang Zenk-Möltgen, GESIS - Leibniz Institute for the Social Sciences 


\section{APPENDIX C}

Copyright (C) DDI Alliance 201 1, All Rights Reserved

\section{http://www.ddialliance.org/}

Content of this document is licensed under a Creative Commons License: Attribution-Noncommercial-Share Alike 3.0 United States

This is a human-readable summary of the Legal Code (the full license).

\section{http://creativecommons.org/licenses/by-nc-sa/3.0/us/}

You are free:

- to Share - to copy, distribute, display, and perform the work

- to Remix - to make derivative works

Under the following conditions:

- Attribution. You must attribute the work in the manner specified by the author or licensor (but not in any way that suggests that they endorse you or your use of the work).

- Noncommercial. You may not use this work for commercial purposes.

- Share Alike. If you alter, transform, or build upon this work, you may distribute the resulting work only under the same or similar license to this one. For any reuse or distribution, you must make clear to others the license terms of this work. The best way to do this is with a link to this Web page.

- Any of the above conditions can be waived if you get permission from the copyright holder.

- Apart from the remix rights granted under this license, nothing in this license impairs or restricts the author's moral rights.

\section{Disclaimer}

The Commons Deed is not a license. It is simply a handy reference for understanding the Legal Code (the full license) - it is a human-readable expression of some of its key terms. Think of it as the user-friendly interface to the Legal Code beneath. This Deed itself has no legal value, and its contents do not appear in the actual license.

Creative Commons is not a law firm and does not provide legal services. Distributing of, displaying of, or linking to this Commons Deed does not create an attorney-client relationship. Your fair use and other rights are in no way affected by the above.

Legal Code:

http://creativecommons.org/licenses/by-nc-sa/3.0/us/legalcode 